\section{IMPROVEMENTS IN RESILIENT WHEELS FOR VEHICLES.}

$\mathrm{A}^{\mathrm{T}}$ a meeting of the Royal Society of Arts held last December, the Hon. $R$. Clere Parsons gave a brief history of the improvements which had been made from

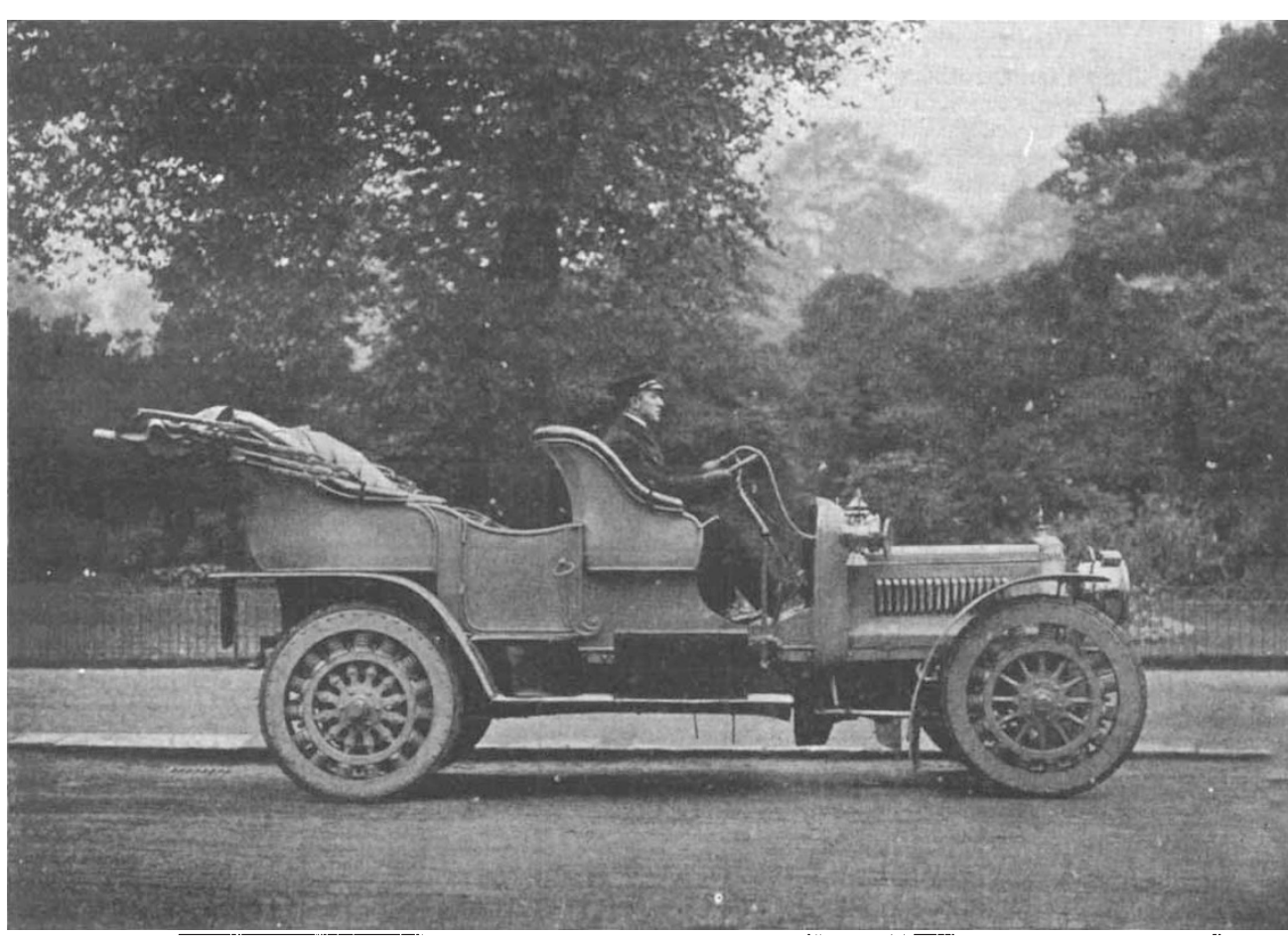

HIG. I. pneumatic tyre, and at

apparatus he described, and in which the effects of the jolts imparted to the wheel were automatically recorded. The result of these investigations, indicated that it was possible to substitute spiral steel springs for the air spring of the pneumatic tyre, and at the same time retain practically all

This special type of wheel, which is known as the "Panflex," from its being flexible in all directions, was then carefully designed, and a set fitted to a $28-36$ horse - power Daimler touring car, illustrated in Fig. I, which in running order weighed 2 tons 5 cwt.

The vheel i 11 ustrated in section and elevation in Figs. 2 and 3 , consists of a steel channel rim similar to those used for solid rubber tyres, and into which a rubber tyre is in serted. On each side of $t h i s$ channel rim are rivetted annular plates. so as to form an interna channel, in the bottom of which a re corrugated time to time in the wheels of vehicles, which had resulted transverse projections. To the wheel centre are rigidly in the extensive adoption of the pneumatic tyre, and then fixed spiral springs at regular intervals, which project passed on to describe a spring wheel which possesses practically all the advantages of a pneumatictyred wheel without its heavy cost of maintenance and liability to puncture. The author explained that, before he attempted to design a spring wheel, he had made a careful search through he records of the British Patent Office Library with the view of ascertaining, if possible, why the numerous inventions relating to this question had not been successful. This search led him to believe that if certain principles were adopted, and careful investigations made, there was a prospect of obtaining a trustworthy spring wheel, which as yet had not been produced.

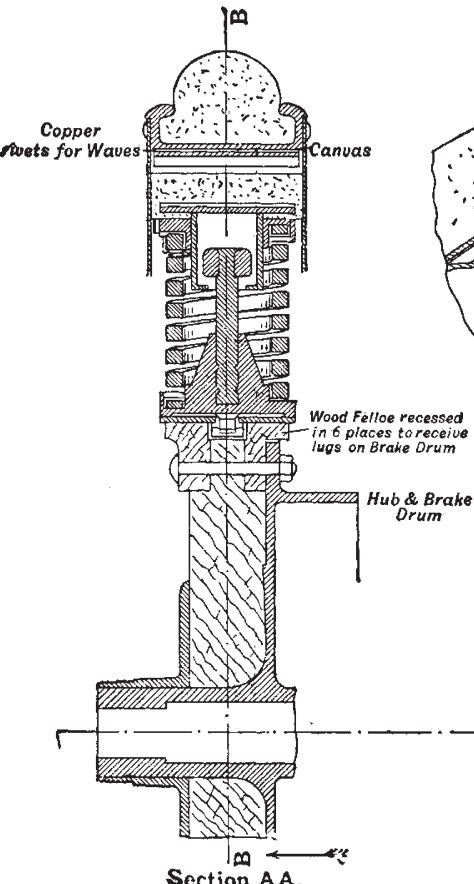

Section AA.

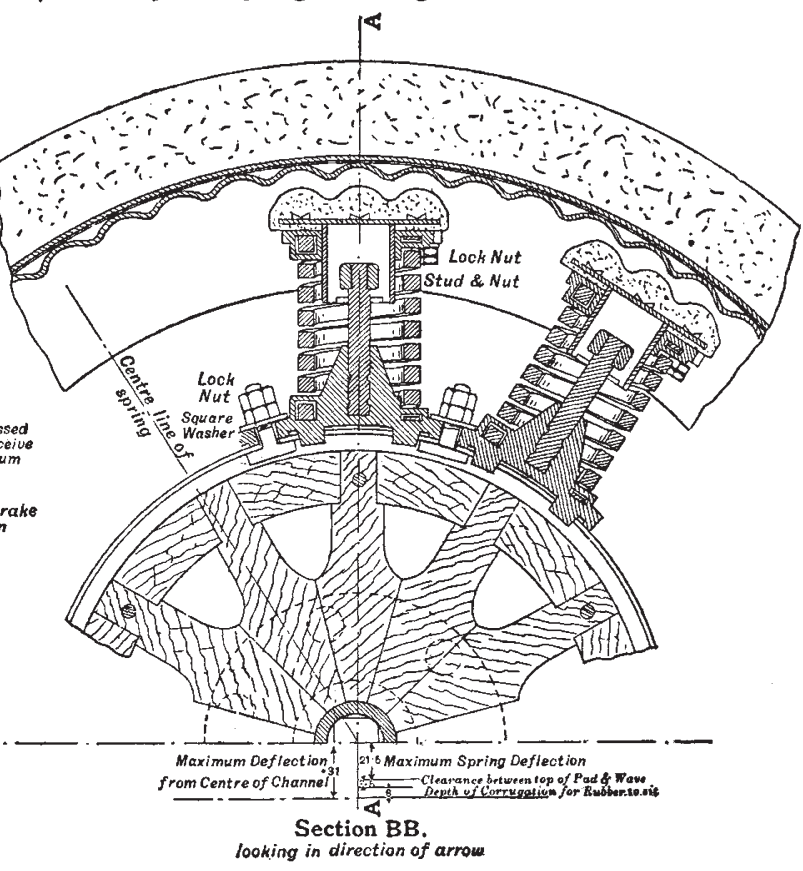

FIG. 3.

The preliminary investigations were made with model / radially outwards, and to their extremities are attached wheels 12 inches in diameter, which were tested in the rubber pads formed with transverse undulations. The NO. $2 \mathrm{IO}_{3}$, VOL. 82] 
diameter of the wheel centre over the pads of the springs being slightly less than that of the channel rim, the wheel centre can be inserted therein, but is not connected in any way with it. The wheel is then complete and ready to be used on a vehicle.

The action of these wheels is simple, and merely consists of the pads on the ends of the spiral springs, as it were, acting as feet, which walk round inside the outer rim, and in turn support the weight of the vehicle. The wheel centre, when the vehicle is travelling along, rotates slightly more rapidly than the outer rim, the advance of the one upon the other being termed the "creep." When any obstacles are passed over, the shocks imparted to the rim of the wheel, which would, if it were a solid one, be transmitted through the axle to the machinery and car body, are absorbed by the rubber pads and springs before they reach the wheel centre. The principle and details of this wheel are quite novel, as has been admitted by the German Patent Office experts previous to granting the patent.

Owing to the rubber caps at the extremities of the spiral springs being capable of deflecting in all directions within certain limits, the friction of the moving parts of the wheel is negligible, and, as a result, practically no heating occurs, saving in maintenance of each car, as regards tyres, by the use of "Panflex" wheels.

In conclusion, the author enumerated the advantages possessed by the "Panflex" wheel, and explained that by its adoption the use of the motor-car could be brought within the reach of persons who are now precluded from availing themselves of it owing to the excessive cost of maintenance of the tyres.

\section{THE SPECTRUM OF THE ZODIACAL LIGHT.}

SINCE the time when Cassini published his observations of the Zodiacal Light, in 1682 , the question as to the nature and origin of this peculiar phenomenon has been constantly recurring. Visual observers were ever at variance on the subject, some holding the view that the Light was a terrestrial adjunct, others that it was a solan appendage. Visually, the matter is a difficult one to decide, for no optical power may be used because of the lack of contrast between the Light and its background of sky. Photography is similarly placed out of court, but it was expected that the question would be solved by the

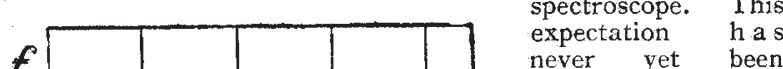
never yet been realised absolutely, but the results recently published (Lick Observatory Bulletin, No. I65) by Dr. Fath clarify matters considerably.

Previous spectroscopic observations have varied on a vital point, viz. the presence or absence of bright radiations in the spectrum, indicating, by their presence, that the Light was selfluminous. Thus some observers found that the spectrum was continuous, others found that its continuity was broken by bright bands, especially the bright aurora line at $\lambda$ 5571. The argument for the pres. ence of the latter as an inherent element of the spectrum was, however, considerably
weakened, if not eliminated, by the observations of weakened, if not eliminated, by the observations of
Respighi, Vogel, Piazzi-Smyth, and others, who were unable to find the aurora line, when observing the spectrum of the Zodiacal Light, unless it was at the same time to be seen in all parts of the sky. Tacchini, Cacciatore, and Ricca similarly were unable to find it, but agreed that the spectrum was continuous, extending from about $\lambda 5000$ to $\lambda 555^{\circ}$, with its maximum intensity at $\lambda 5350$; that is to say, the spectrum of the Light resembled the solar spectrum without the absorption lines.

The detection of the dark solar lines is a difficulty inherent to the observation, for the Light is so faint that, to get a visible spectrum, a broad slit is essential, and with a broad slit the comparatively fine absorption lines are lost.

Wright, in 1874 , was able to use a narrower slit, and found that the spectrum, with an intensity-curve similar to that of daylight, showed traces of an absorption band in the position of the $\delta$ band in the solar spectrum.

Thus it became fairly evident that the Zodiacal Light was reflected sunlight, and the opinion now generally held is that it is reflected by a collection of small meteoritic

maintenance is No. 2103 , VOL. 82] 